\title{
More effective use $=$ more lives saved: medical countermeasure response strategies for mass casualty radiological incidents.
}

\author{
Juanita Jones ${ }^{1}$, and Chad M. Hrdina ${ }^{1}$ \\ ${ }^{1}$ Assistant Secretary for Preparedness and Response, United States Department of Health and Human \\ Services, 200 Independent Avenue SW, Washington, DC 20001, United States of America \\ Topic: medical countermeasures and decorporation
}

\section{Introduction}

Strategies for the use of medical countermeasures are vital to ensure maximum lifesaving during an incident response. Federal budgets for development and acquisition of medical countermeasures (MCMs) to chemical, biologic, radiological and nuclear (CBRN) threats are often inadequate to fund infinite sustainment of national stockpiles. In addition, necessary infrastructure, diagnostic capabilities, operational, and personnel resources can also be limited or unavailable due to lack of technology or inadequate funds for investment. Thus, federal governments and community emergency medical response and public health partners must develop strategies to leverage scarce MCMs and other resources to ensure a mass-casualty response approach to a CBRN incident that efficiently and effectively saves the most lives. The decision framework approach and MCM response strategies for radiological incidents will be presented.

\section{Strategic Framework}

Medical countermeasure response strategies are divided into three major portions with supporting information: 1) Notification, and 2) Scope of incident, lead to 3) an MCM response strategy, which is supported with MCM specific information, special considerations for special populations (e.g., children), regulatory or legal issues or needs for MCM utilization, as well as useful public messages recommendations, and current clinical guidelines summary.

\subsection{Notification and incident scope}

How we become aware of an incident is an indicator as to the potential affected population and severity of injuries or illness at the time of notification, which means we can anticipate the spectrum of casualties resulting from the incident, and make decisions for allocation for MCMs such as Prussian blue or Ca/Zn-DTPA. The nature of an incident, including factors such as time, affected population, ability to identify affected population, mode and magnitude of radiological dispersal are important because they allow responders and decision makers to identify and characterize the population that has been exposed, or at risk 
of exposure. This informs decisions to protect a population at risk, from any or further exposure, and to implement public health or medical interventions to mitigate or treat injury from radiation exposure or internal contamination with radionuclides. A very important aspect of characterizing the magnitude of an incident translates into an estimation as to whether enough resources are available or there is potential for a shortage. Scoping the incident is not about identifying all of the potential scenarios, but rather those factors that differentiate scenarios, such that one would change the strategic approach to response.

\subsection{MCM response strategy}

In the initial response period, much situational information many not be known, but decisions will be required to ensure assets, including medical countermeasures are quickly employed early. As more information becomes available, flexible decision makers can adjust the medical countermeasure response approach. The MCM response strategy discusses target populations and intended population-based use of medical countermeasures. Deployment and distribution of medical countermeasures may include strategies for prophylaxis, mitigation, treatment, of target populations with different medical countermeasures at different times. The MCM response strategy recommendations ensure efficient and effective use of medical countermeasures based on confirmed or estimated factors of the incident and address both adequate and scarce resource strategies.

\section{Radiological incident MCM response strategy}

Potential for scarce resources following a radiological incident is an important issue that requires consideration for $\mathrm{MCM}$ distribution strategies. We recommend that in a scarce resources situation, early decorporation remains an important mitigation step that should not be delayed for confirmatory bioassay to initiate therapy as a means to conserve MCM resources. Instead, our analyses support an approach where epidemiology and simple screening can inform pre-emptive administration of MCMs to casualties with suspected internal contamination, which can save more lives than bioassay confirmation as the initiation threshold for decorporation. We recommend bioassay for discontinuation of therapy, and there may be dosing alternatives, as well as alternative thresholds for discontinuation of therapy, to conserve medical countermeasures. Furthermore, conservation of resources means establishing appropriate thresholds for target populations, which could mean palliative care for those too sick to benefit from decorporation.

Likewise, response to an incident with sufficient resources availability for the internally contaminated population should also leverage bioassay for discontinuation of therapy where thresholds might be set far lower, and screening may include those with less risk. Regardless of resource levels, bioassay is should be prioritized in cases where confirmation is preferred before initiation of pre-emptive therapy, to reduce high risk for adverse effects of unnecessary MCM administration (e.g. neonates).

\section{Conclusion}

Deliberative planning the strategic use of medical countermeasures for a variety of radiological risks means that if a radiological incident were to occur, we are prepared to use medical countermeasures in the most efficient ways, with the most effective approaches, such that we maximize the lives-saved. Planning prevents lengthy contemplation during 
response that could impede timely access to medical countermeasures by those who need them. 